\title{
2S Modules for the Phase-2 Upgrade of the CMS Tracker
}

Oliver Pooth*广

RWTH Aachen University

E-mail: pooth@physik.rwth-aachen.de

The CMS experiment at the Large Hadron Collider LHC will face a new era after Long Shutdown 3, which is scheduled to take place between 2024 and 2026. The challenge of running a tracking detector at the High Luminosity LHC will be the high instantaneous luminosity of $5 \times 10^{34} \mathrm{~s}^{-1} \mathrm{~cm}^{-2}$, leading to a total integrated luminosity of $3000 \mathrm{fb}^{-1}$. Due to the high instantaneous luminosity a particle flux of up to $1 \times 10^{15} \mathrm{n}_{\mathrm{eq}} \mathrm{cm}^{-2}$ is expected in the outer tracker region. To cope with this challenge the existing CMS Outer Tracker has to be replaced by a completely new subdetector system. This Phase- 2 tracker will have an increased granularity with $\leq 1 \%$ occupancy in all regions and less material compared to the current detector. In addition the CMS Phase-2 tracker will contribute to the level-1 trigger with specialized detector modules with onboard transverse momentum discrimination. The Phase-2 Outer Tracker will consist of 5616 PS modules with a macro-pixel and a strip sensor on top of each other, and $76802 \mathrm{~S}$ modules with two strip sensors on top of each other. In the next years standardized production lines for precise module building and testing are going to be installed in all $2 \mathrm{~S}$ module production centers in the USA, Germany, Belgium, Pakistan, and India. The current plan foresees to integrate PS and 2S modules on tracker substructures starting 2022 and to start integrating the complete Phase-2 tracker in CMS in 2024 for the operation at the High Luminosity LHC.

The 39th International Conference on High Energy Physics (ICHEP2018)

4-11 July, 2018

Seoul, Korea

* Speaker.

${ }^{\dagger}$ on behalf of the CMS Tracker Group 


\section{2S Modules}

The $2 \mathrm{~S}$ modules (Figure 1) are built from two silicon strip sensors with a distance of $1.8 \mathrm{~mm}$ or $4 \mathrm{~mm}$, depending on the region in the detector. Each sensor contains $2 \times 1016$ strips with a length of $5 \mathrm{~cm}$ and a pitch of $90 \mu \mathrm{m}$. The active area of approximately $92 \mathrm{~cm}^{2}$ on the $10 \times 10 \mathrm{~cm}^{2}$ sensor is read out by sixteen $\mathrm{CBC} 3$ front-end chips forming hit pairs from the two sensors. Power distribution, controls, and data readout are realized via service hybrids at the side of the module. The power consumption is $5.0 \mathrm{~W}$ for the front-end electronics and $1.0 \mathrm{~W}$ for the sensors at $-20^{\circ} \mathrm{C}$.
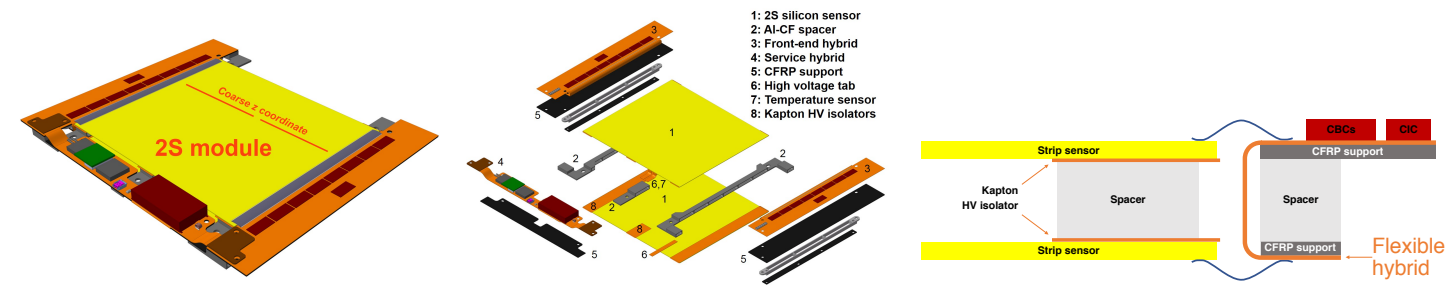

Figure 1: 2S module [1]. Left: view of the assembled modules. Center: details of the module parts. Right: cross section of the module and front-end hybrid folded assembly and connectivity.

\section{Level-1 Trigger with Tracker Information}

On the PS and 2S modules the $\mathrm{CBC} 3$ front-end chips correlate the signals from the two sensor planes and select pairs that form so-called stubs compatible with a particle above an adjustable transverse momentum threshold. These stubs are used to form tracks and the CMS Track Finder sends the track parameters with $40 \mathrm{MHz}$ to the level-1 trigger, where the information is combined with the calorimeter and muon triggers. With up to $750 \mathrm{kHz}$ the information is passed from the level-1 trigger to the CMS high-level trigger for further data reduction and final readout.

\section{2S Module Production Line}

In a production center for CMS $2 \mathrm{~S}$ modules several steps are necessary for module building and testing prior to module integration on larger tracker substructures. After reception tests of all parts, glueing steps are done to connect high voltage Kapton strips to the silicon sensors. The bare $2 \mathrm{~S}$ module is built on a mechanical precision jig out of sensors, aluminium/carbon fiber reinforced distance bridges, front-end hybrids, and service hybrids. The mechanical assembly precision of the sensors must be better than $50 \mu \mathrm{m}$ perpendicular to the strips and $100 \mu \mathrm{m}$ parallel to the strips, with a tilt angle below $400 \mu \mathrm{rad}$. The precision is checked using a metrology machine. Wire bonding and wire bond encapsulation finalizes the $2 \mathrm{~S}$ modules before a module burn-in test qualifies the modules for the integration on larger substructures. The first functional modules with non-final components have been built in 2018 within mechanical specifications.

\section{References}

[1] The Phase-2 Upgrade of the CMS Tracker, CERN-LHCC-2017-009 ; CMS-TDR-014. 